MATEC Web of Conferences 48,03006 (2016)

DOI: $10.1051 /$ matecconf $/ 20164803006$

(C) Owned by the authors, published by EDP Sciences, 2016

\title{
Small-angle tomography algorithm for transmission inspection of acoustic linear array
}

\author{
Alexey Soldatov ${ }^{1}$, a , Andrey Soldatov ${ }^{1}$, Masha Kostina ${ }^{1}$, Petr Bakanov ${ }^{2}$ and Marina Polonskaya ${ }^{1}$ \\ ${ }^{1}$ National Research Tomsk Polytechnic University, 634050 Tomsk, Russia \\ ${ }^{2}$ Ultrasonic technology Ltd., 634027 Urozhayniy bystreet, Russia
}

\begin{abstract}
The paper describes the algorithm of reconstruction of tomographic image used in the through-transition method in a small angle sounding of acoustic linear arrays and the results of practical application of the proposed algorithm. In alternate probing of each element of emitting array and simultaneous reception of all elements of the receiving array is a collection of shadow images of the testing zone. The testing zone is divided into small local areas and using the collection of shadow images computed matrix normalized transmission coefficients for each of the small local area. Tomographic image control zone is obtained by submitting the resulting matrix of normalized transmission coefficients in grayscale or colors.
\end{abstract}

\section{Introduction}

Through-transition method has a higher sensitivity and noise immunity, and also has no dead area, in comparison with the pulse-echo method. This method is particularly effective in the testing of the multi-layer composites are widely used at present in aircraft and aerospace [1]. Traditionally, two transducers, emitter and receiver, are used in this method. They are arranged on the opposite sides of the testing object $[2,3]$. But, to use only two sensors will not make it possible to determine the distance to the defect [4]. In order to calculate the distance to the defect one must have the projections from different angles, as it is implemented in the computed tomography, which has been widely developed in recent years [5]. It is possible to use two different methods of the acoustic testing: mechanical scanning of the testing object with two sensors - a receiving and emitting, with its position that is fractional to the object testing by discrete and independent of each other, or an electronic scanning of the testing object with two linear arrays.

The advantage of the first option - the possibility of setting an arbitrary step and tracing.

Disadvantages: - long time of the scanning, because of the mechanical sensors travel, - the complicacy and high cost of high-precision mechanical scanner with a programmable control, - the need for high stability of measuring system.

The advantage of the second option - possibility to have a high-speed scanning, but only under such condition - with a fixed geometrical structure of a concrete device. Disadvantage: need of a high stability of equipment when sensor of antenna arrays is emitted.

\footnotetext{
a Corresponding author : asoldatof@mail.ru
} 


\section{MATEC Web of Conferences}

\section{Problem statement}

In practice, the electronic scanning is used. It should be noted that when the acoustic testing is used it is impossible to obtain a collection of projection angles from 0 to 360 degrees. When linear arrays are had the electronic scanning we can have a limited number of projections with a small angular deviation, under the testing of planar structures such as multilayer composites as a rule, no more than \pm 30 degrees $[6,7]$. Firstly, limitation is linked with the need, in order to provide access from different sides, and secondly, to provide an acoustical coupling sensor with the object of testing. Therefore, it is of interest to study the possibilities of small-angle sensing. For this purpose it is proposed 2D-model of acoustic through-transition detection system with linear arrays and logical and analytical image reconstruction algorithm (visualization) of the internal structure of the object in the testing zone.

\section{Two-dimensional model}

A two-dimensional model of the acoustic patch with the emitting and receiving arrays is shown in Figure 1.

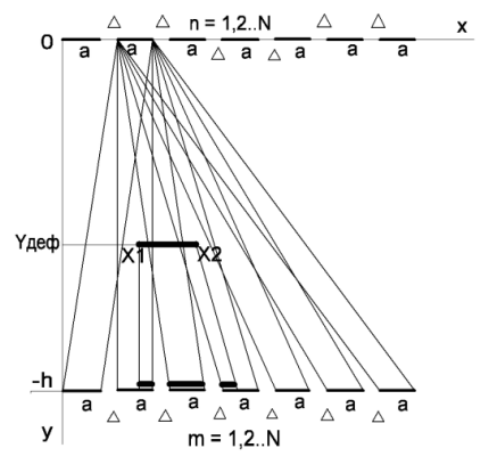

Figure 1. The acoustic path of through-transition method with linear arrays.

Each acoustic arrays consists of $N$ elements with sizes of element "a" equal to the actual diameter of cylindrical piezoelectric element and placed at the same distance $\Delta$ from each other. The latter condition is not fundamentally necessary for a real system and introduced only for the convenience of the analytical description of the model. Antenna arrays, dispose on the opposite sides of the testing zone, located collinear with the $x$-axis at a distance $h$ from one another. Thus, the area of the testing zone is:

$$
S=[(a+\Delta) N-\Delta] h,
$$

where $a$ - the size of the piezoelectric element, $\Delta$ - the distance between the piezoelectric element, $N$ - number of elements in the array, $h$ - the distance between the antenna arrays.

Sounding of the testing zone is carried out by means of alternately switching of the emitting sensors. All these elements of the receiver array are recorded by signal that has been passed through the testing object [8]. The result of monitoring procedures is the transmission coefficient matrix of size $(N * N)$. The coefficients are calculated in accordance with the formula:

$$
k_{m, n}=A_{m, n} / A_{0},
$$

where $A_{m, n}$ - the signal amplitude at the receiver $m$ when the transmitter $n$ shots and the object has the defect, $A_{0}$ - the signal amplitude at the receiver $m$ when the transmitter $n$ shots and the object doesn't have the defect. 
When the testing object doesn't have the defects then the coefficient matrix of transmission is an unitary matrix. It is assumed that elements of the transmitter and receiver are isotropic in the model, the medium loss equals zero, the radiation from $n$ element of the emitting array into $m$ element of the receiving array is equivalent of geometrical acoustics within the zone (radiation tape), formed by straight lines connecting the beginning of the radiation element to the beginning of the receiver element and the end of the radiation element to the end of the receiving element, and diffraction is ignored. (The concept of radiation tape was introduced for 2D-model and analogical with the meaning of Williams tube (lightpipe) for 3D-model). Zone borders described by the equations of straight lines [9], which are convenient to represent, in accordance with, in the form:

$$
\begin{aligned}
& \text { the left (odd) boundary } \quad \frac{x-x l(n)}{x 2(m)-x l(n)}=\frac{y-y l(n)}{y 2(m)-y 1(n)}, \\
& \text { the right (even) boundary } \frac{x-(x 1(n)+a)}{(x 2(m)+a)-(x 1(n)+a)}=\frac{y-y 1(n)}{y 2(m)-y 1(n)},
\end{aligned}
$$

where $x 1(n)$ - the left end $n$-th element of radiating array $(n=1,2 . . N), x 2(m)$ - the left end of the $m$ order element of receiving array $(m=1,2 . . N), y 1(n)$ and $y 2(m)$ - the coordinates of the radiating and receiving elements arrays. They are easy to take equal $y 1(n)=0$ and $y 2(m)=-\mathrm{h}$.

A defect in the model is simulated by a straight line segment that is parallel to the $x$ axis, and it is located in the testing zone with the coordinates $X 1, X 2, Y$. The defect supposed to be opaque. The defect attenuation depends on the overlap of radiation tape (Figure 2). Therefore, the transmission ratios are calculated in accordance with formula [10]:

$$
k_{m, n}=1-S_{m, n} / S_{0}
$$

where $S_{m, n}$ - the shadow length from the defect on the receiver $m$ for angle of the emitter $n, S_{0}$ - the length of the receiver.

If there is not defect in the radiation tape, then $k_{m, n}=1$. If the defect covers the radiation tape on the half, then $k_{m, n}=0.5$.

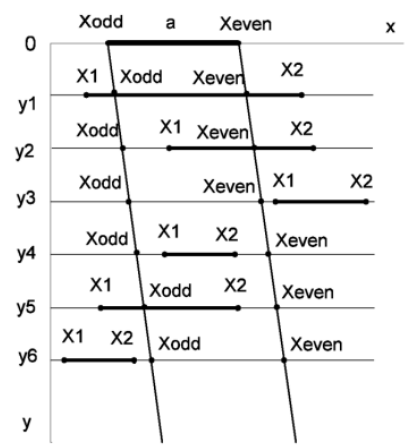

Figure 2. Options of the defects location in the testing zone, $X 1, X 2$ - coordinates of the defect boundaries, $x_{o d d}$, $x_{\text {even }}$ - coordinates of the radiation boundaries tape at different level $\mathrm{Ydef}(\mathrm{Y} 1, \mathrm{Y} 2$ and etc.).

Options of the possible defect location relatively to the radiation tape and the corresponding transmission coefficients are summarized in Table 1. 


\section{MATEC Web of Conferences}

Table 1. Options of the possible defect location.

\begin{tabular}{|c|c|}
\hline Conditions characterizing the defect placement in the testing zone & transmission factor $k_{m, n}$ \\
\hline$X 1 \leq x_{\text {odd }}(n, m)<x_{\text {even }}(n, m) \leq X 2$ & 0 \\
\hline$x_{\text {odd }}(n, m)<X 1<x_{\text {even }}(n, m) \leq X 2$ & $0<X 1-x_{\text {odd }}(n, m) / a<1$ \\
\hline$x_{\text {odd }}(n, m)<x_{\text {even }}(n, m) \leq X 1<X 2$ & 1 \\
\hline$x_{\text {odd }}(n, m)<X 1<X 2<x_{\text {even }}(n, m)$ & $0<a-(X 2-X 1) / a<1$ \\
\hline$X 1<x_{\text {odd }}(n, m)<X 2<x_{\text {even }}(n, m)$ & $0<x_{\text {even }}(n, m)-X 2 / a<1$ \\
\hline$X 1<X 2 \leq x_{\text {odd }}(n, m)<x_{\text {even }}(n, m)$ & 1 \\
\hline
\end{tabular}

A matrix of $N^{*} N$ transmission coefficients $k_{m, n}$ (Figure 3) was given as an example of simulation results. The results were obtained with linear radiating and receiving arrays located at distance $h=60 \mathrm{~mm}$, each array consists of $N=8$ elements with size $a=6 \mathrm{~mm}$ and distance between elements is $\Delta=3 \mathrm{~mm}$. Defect coordinates are $X 1=32.1 \mathrm{~mm}, X 2=37.1 \mathrm{~mm}$ and $Y$ def $=-40 \mathrm{~mm}$.

$$
A=\left(\begin{array}{cccccccc}
1 & 1 & 1 & 1 & 1 & 1 & 0.85 & 0.167 \\
1 & 1 & 1 & 1 & 1 & 0.85 & 0.167 & 0.817 \\
1 & 1 & 1 & 1 & 0.85 & 0.167 & 0.817 & 1 \\
1 & 1 & 1 & 0.85 & 0.167 & 0.817 & 1 & 1 \\
1 & 1 & 0.85 & 0.167 & 0.817 & 1 & 1 & 1 \\
1 & 0.85 & 0.167 & 0.817 & 1 & 1 & 1 & 1 \\
0.85 & 0.167 & 0.817 & 1 & 1 & 1 & 1 & 1 \\
0.167 & 0.817 & 1 & 1 & 1 & 1 & 1 & 1
\end{array}\right)
$$

Figure 3. Matrix of coefficients $k_{m, n}$, when the location of defect coordinates are $X 1=32.1 \mathrm{~mm}, X 2=37.1 \mathrm{~mm}$ and $Y \mathrm{def}=-40 \mathrm{~mm}$.

A graphical matrix representation of transmission coefficients $k_{m, n}$ in grayscale is shown in figure 4 .

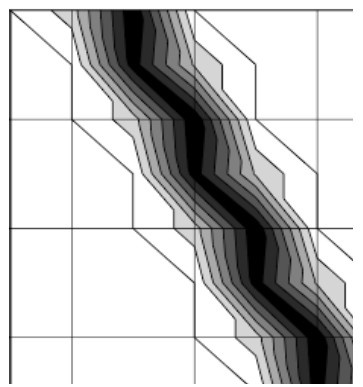

Figure 4. A graphical matrix representation of the coefficients $k_{m, n}$.

Analysis of a graphical matrix representation of the transmission coefficients $k_{m, n}$ is shown, if the testing zone has a defect then it is possible:

- determine the position of the defect on the slope of the line, representing a linear approximation of the location of the minima of the transmission coefficients $k_{m, n}$ in the matrix rows, for example, performed by the least square method,

- make an assessment of the size of the defect and its transfer properties by the transmission coefficients value $k_{m, n}$,

- it can be inferred by the linear dimensions on the bandwidth of the minima defect of the matrix coefficients $k_{m, n}$ in the graph. 


\section{Calculation of the matrix transmission coefficients}

The greatest interest is the investigation of the possibility to evaluate the state of the testing object at each point of the testing zone when one use the transmission matrix coefficients is obtained, in this case, with linear acoustic arrays that is equivalent to obtain the tomographic image of the testing zone. The matrix transmission coefficients for the model with acoustic linear arrays from $N$ elements each represents $(2 N-1)$ truncated collections of radiation projection, transmitted through the testing zone to the receiving array at angles from $0^{\circ}$ to $\pm \varphi(k)$ :

$$
\varphi(k)=\operatorname{arctg}(k(a+\Delta) / h),
$$

where $k=0,1 . .(N-1)$.

Moreover, if collections of projections for $k=0$ is equal to $N$ (the only complete), for $k=1$ is equal to $(N-1)$ and so on, then for $k=(N-1)$, it is equal only to one (unitary) [11].

It is impossible to use Radon's transformation [12] for visualization because we have a truncated collections of projections from emitters to receivers that probe the testing zone at different angles. In this regard, for the reconstruction of tomographic image of the testing zone the algoritm was proposed and its modeling was carried out in Mathcad .The algorithm is based on the following principles:

- testing zone is divided into small local areas that are considered to be point,

- every point of space in the testing zone (between the emitting and receiving arrays) because of its abnormality (defects) has the affect on the transmission coefficients $k_{m, n}$. Thus each specific point affects to the value $k_{m, n}$ of those radiation tapes, where the point comes to these fields,

- the resulting attenuation characterized by abnormal of this concrete point as it was supposed to define as the sum of the transmission coefficients $k_{x, y}=\sum_{1}^{P(x, y)} k_{m, n} / P(x, y)$, divided into the number of radiation tapes that were passed through this point (normalized transfer coefficient for a point with coordinates $x$ and $y$ ),

- if even one or more of the coefficient $k_{m, n}$ of radiation tapes, in which the given point came, occurs equal to one (transmission in the radiation tape is maximum in this case), then the resulting normalized sums of $k_{x, y}$, for the given point, it should be taken up as equal to one, because this point of space does not inject the losses (correcting condition).

The structure of tomography reconstruction algorithm is shown in figure 5 .

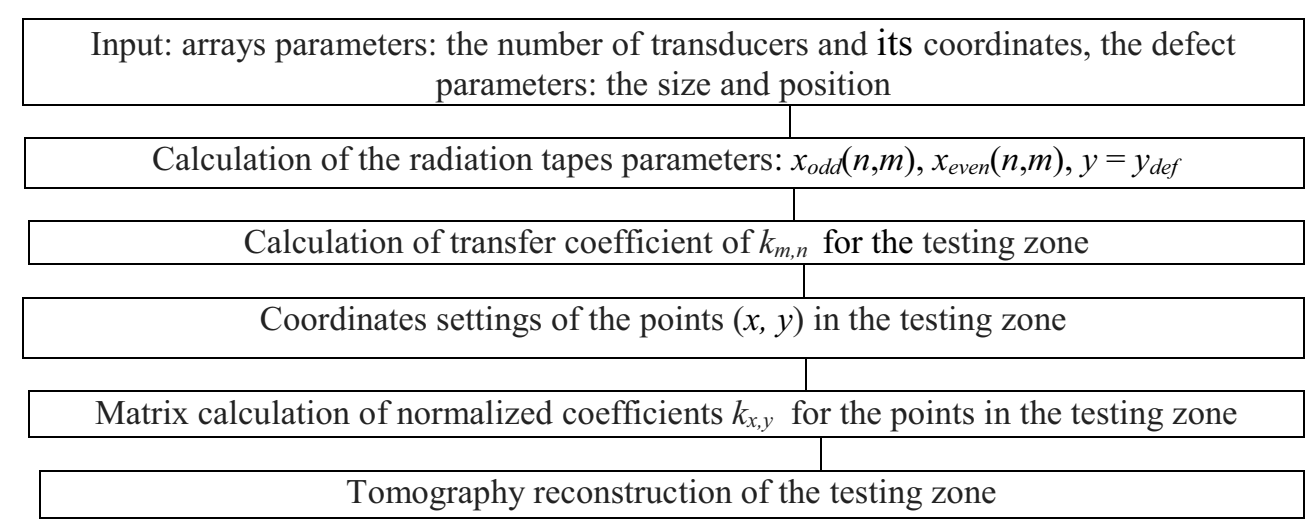

Figure 5. Tomography reconstruction algorithm. 


\section{MATEC Web of Conferences}

The calculation result of the matrix transmission coefficients $k_{x, y}$ in the testing zone in the proposed algorithm on the dates analogical with figure 4 , shown in figure 6 . The point of space in the testing zone were set at a distance of $2.5 \mathrm{~mm}$ from one another in the coordinates of $\langle x\rangle$ and $\langle y\rangle$.

\begin{tabular}{|c|c|c|c|c|c|c|c|c|c|c|c|c|c|c|c|c|c|c|c|c|c|}
\hline 0.979 & 1 & 1 & 1 & 1 & 1 & 1 & 1 & 1 & 1 & 1 & 1 & 1 & 1 & 1 & 1 & 1 & 1 & 1 & 1 & 1 & 1 \\
\hline 0.877 & 0.979 & 1 & 1 & 1 & 1 & 1 & 1 & 1 & 1 & 1 & 1 & 1 & 1 & 1 & 1 & 1 & 1 & 1 & 1 & 1 & 0.979 \\
\hline 0.754 & 0.836 & 0.97 & 1 & 1 & 1 & 1 & 1 & 1 & 1 & 1 & 1 & 1 & 1 & 1 & 1 & 1 & 1 & 1 & 1 & 0.97 & 0.836 \\
\hline 1 & 0.803 & 0.836 & 0.975 & 1 & 1 & 1 & 1 & 1 & 1 & 1 & 1 & 1 & 1 & 1 & 1 & 1 & 1 & 1 & 0.975 & 0.836 & 0.803 \\
\hline 0.854 & 0.975 & 0.86 & 0.86 & 0.975 & 1 & 1 & 1 & 1 & 1 & 1 & 1 & 1 & 1 & 1 & 1 & 1 & 1 & 0.975 & 0.86 & 0.86 & 0.975 \\
\hline 0.833 & 0.833 & 0.836 & 0.858 & 0.877 & 0.979 & 1 & 1 & 1 & 1 & 1 & 1 & 1 & 1 & 1 & 1 & 1 & 0.979 & 0.877 & 0.858 & 0.836 & 0.833 \\
\hline 1 & 0.767 & 0.806 & 0.836 & 0.836 & 0.836 & 0.979 & 1 & 1 & 1 & 1 & 1 & 1 & 1 & 1 & 1 & 0.979 & 0.836 & 0.836 & 0.836 & 0.806 & 0.767 \\
\hline 0.854 & 0.97 & 0.831 & 0.833 & 0.86 & 0.803 & 0.858 & 0.979 & 1 & 1 & 1 & 1 & 1 & 1 & 1 & 0.979 & 0.858 & 0.803 & 0.86 & 0.833 & 0.831 & 0.97 \\
\hline 0.854 & 0.854 & 0.806 & 0.75 & 0.812 & 0.835 & 0.773 & 0.858 & 0.967 & 0.981 & 1 & 1 & 1 & 0.981 & 0.967 & 0.858 & 0.773 & 0.835 & 0.812 & 0.75 & 0.806 & 0.854 \\
\hline 0.661 & 0.767 & 0.833 & 0.806 & 0.806 & 0.75 & 0.835 & 0.754 & 0.857 & 0.963 & 0.981 & 1 & 0.981 & 0.963 & 0.857 & 0.754 & 0.835 & 0.75 & 0.806 & 0.806 & 0.833 & 0.767 \\
\hline 0.803 & 0.97 & 0.944 & 0.833 & 0.812 & 0.813 & 0.731 & 0.731 & 0.735 & 0.841 & 0.925 & 0.919 & 0.925 & 0.841 & 0.735 & 0.731 & 0.731 & 0.813 & 0.812 & 0.833 & 0.944 & 0.97 \\
\hline 0.854 & 0.854 & 0.767 & 0.831 & 0.806 & 0.75 & 0.727 & 0.724 & 0.713 & 0.675 & 0.728 & 0.85 & 0.728 & 0.675 & 0.713 & 0.724 & 0.727 & 0.75 & 0.806 & 0.831 & 0.767 & 0.854 \\
\hline 0.806 & 0.767 & 0.806 & 0.806 & 0.812 & 0.813 & 0.813 & 0.708 & 0.69 & 0.543 & 0.47 & 0.486 & 0.47 & 0.543 & 0.69 & 0.708 & 0.813 & 0.813 & 0.812 & 0.806 & 0.806 & 0.767 \\
\hline 1 & 0.97 & 0.833 & 0.833 & 0.806 & 0.75 & 0.731 & 0.708 & 0.65 & 0.535 & 0.365 & 0.167 & 0.365 & 0.535 & 0.65 & 0.708 & 0.731 & 0.75 & 0.806 & 0.833 & 0.833 & 0.97 \\
\hline 0.854 & 0.833 & 0.833 & 0.854 & 0.812 & 0.813 & 0.727 & 0.708 & 0.665 & 0.6 & 0.557 & 0.47 & 0.557 & 0.6 & 0.665 & 0.708 & 0.727 & 0.813 & 0.812 & 0.854 & 0.833 & 0.833 \\
\hline 0.854 & 0.806 & 0.831 & 0.806 & 0.75 & 0.767 & 0.813 & 0.727 & 0.642 & 0.733 & 0.785 & 0.817 & 0.785 & 0.733 & 0.642 & 0.727 & 0.813 & 0.767 & 0.75 & 0.806 & 0.831 & 0.806 \\
\hline 1 & 0.97 & 0.836 & 0.833 & 0.806 & 0.767 & 0.75 & 0.746 & 0.746 & 0.846 & 0.939 & 1 & 0.939 & 0.846 & 0.746 & 0.746 & 0.75 & 0.767 & 0.806 & 0.833 & 0.836 & 0.97 \\
\hline 0.806 & 0.806 & 0.833 & 0.835 & 0.75 & 0.831 & 0.746 & 0.815 & 0.867 & 0.959 & 1 & 1 & 1 & 0.959 & 0.867 & 0.815 & 0.746 & 0.831 & 0.75 & 0.835 & 0.833 & 0.806 \\
\hline 0.854 & 0.833 & 0.833 & 0.806 & 0.833 & 0.769 & 0.829 & 0.85 & 0.977 & 1 & 1 & 1 & 1 & 1 & 0.977 & 0.85 & 0.829 & 0.769 & 0.833 & 0.806 & 0.833 & 0.833 \\
\hline 0.963 & 0.97 & 0.806 & 0.833 & 0.736 & 0.85 & 0.855 & 0.974 & 1 & 1 & 1 & 1 & 1 & 1 & 1 & 0.974 & 0.855 & 0.85 & 0.736 & 0.833 & 0.806 & 0.97 \\
\hline 0.611 & 0.806 & 0.806 & 0.769 & 0.85 & 0.873 & 0.974 & 1 & 1 & 1 & 1 & 1 & 1 & 1 & 1 & 1 & 0.974 & 0.873 & 0.85 & 0.769 & 0.806 & 0.806 \\
\hline 0.854 & 0.854 & 0.963 & 0.831 & 0.831 & 0.974 & 1 & 1 & 1 & 1 & 1 & 1 & 1 & 1 & 1 & 1 & 1 & 0.974 & 0.831 & 0.831 & 0.963 & 0.854 \\
\hline 0.854 & 1 & 0.855 & 0.855 & 0.974 & 1 & 1 & 1 & 1 & 1 & 1 & 1 & 1 & 1 & 1 & 1 & 1 & 1 & 0.974 & 0.855 & 0.855 & 1 \\
\hline 1 & 0.831 & 0.855 & 0.974 & 1 & 1 & 1 & 1 & 1 & 1 & 1 & 1 & 1 & 1 & 1 & 1 & 1 & 1 & 1 & 0.974 & 0.855 & 0.831 \\
\hline 0.831 & 0.855 & 1 & 1 & 1 & 1 & 1 & 1 & 1 & 1 & 1 & 1 & 1 & 1 & 1 & 1 & 1 & 1 & 1 & 1 & 1 & 0.855 \\
\hline 0.873 & 1 & 1 & 1 & 1 & 1 & 1 & 1 & 1 & 1 & 1 & 1 & 1 & 1 & 1 & 1 & 1 & 1 & 1 & 1 & 1 & 1 \\
\hline
\end{tabular}

Figure 6. Matrix of the distribution of the resulting transfer coefficients of $k_{x, y}$ in the testing zone with the location of the defect with coordinates $X 1=32.1 \mathrm{~mm}, X 2=37.1 \mathrm{~mm}, Y$ def $=-40 \mathrm{~mm}$.

\section{Results}

If each value in the normalized coefficient matrix to represent as grayscales, we will obtain a tomographic image of the testing zone (figure 7).

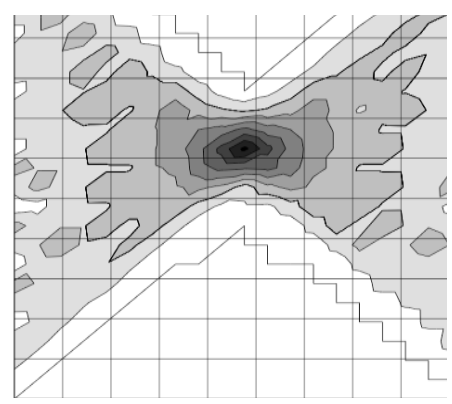

Figure 7. Tomographic image of the testing zone, corresponds to the normalized coefficient matrix.

Result shown in figure 7 was obtained without using the correction of the conditions: if even one or more of the coefficient $k_{m, n}$ of radiation tapes, which belongs to this point, it is equal to one, then $k_{x, y}$, passed through this point is equal to one. 
Figure 8 shows the imaging testing zone with the defect with coordinates $X 1=17,1 \mathrm{~mm}$, $X 2=25,1 \mathrm{~mm}$ and ydef $=-30 \mathrm{~mm}$ without using the conditions correction (a) and with its using (b) for $N=4, a=6 \mathrm{~mm}, \Delta=3 \mathrm{~mm}, h=60 \mathrm{~mm}$, the distance between the points in the testing zone is $5 \mathrm{~mm}$ with total number of points $\mathrm{M}=66(100 \%)$. Thus, if in the first case we record as the abnormal points $53(80.3 \%)$, while in the second case, only $18(27.7 \%)$, this increase the localization of the defect position significantly. However, the area of the minimum values of the coefficient $k_{x, y}$ remained unchanged.

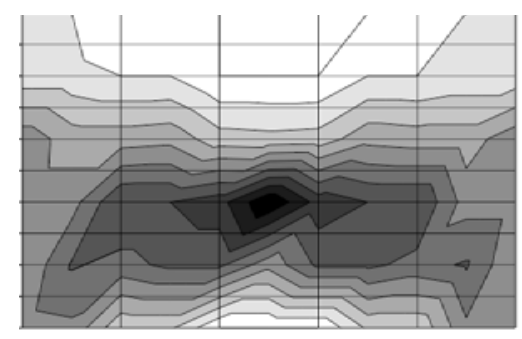

a

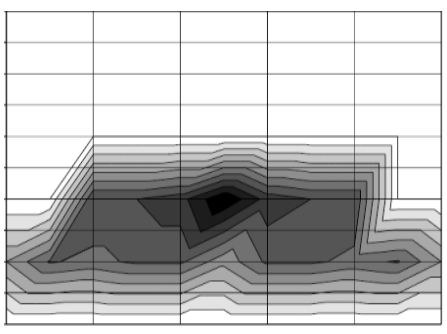

$\mathrm{b}$

Figure 8. Tomographic image of testing zone without correction (a), and with correction (b), for $N=4$, $a=6 \mathrm{~mm}, \Delta=3 \mathrm{~mm}, h=60 \mathrm{~mm}$, the coordinates of the defect $X 1=17,1 \mathrm{~mm}, X 2=25,1 \mathrm{~mm}$ and $Y$ def $=-30 \mathrm{~mm}$.

\section{Conclusion}

The performed simulations have shown the possibility of tomographic reconstruction of the testing zone at a small-angle sensing with linear acoustic arrays use. The algorithm allows you to specify the number of local points in the testing zone, the parameters of acoustic arrays: the number of emitting and receiving elements, the distance between them, as well as the distance between the arrays. Application of the proposed algorithm of tomography reconstruction of the testing zone allows to localize the spatial position of the defect with an error of no more than $10 \%$ of the size of the testing zone. The change of the local areas number in the testing zone, with the fixed parameters of the acoustic path, does not significantly increase the accuracy of the localization of the spatial position of the defect. It is possible to increase the accuracy by means of increasing the number of angles and angular deflection angles that is achieved by means of increasing the number of elements in the arrays.

\section{References}

1. A. Tovstik, A. Zakarluka, A. Petrenko, Herald of the Dnepropetrovsk University 17, 96 (2014)

2. R. Rausutis, A. Voleisis, R. Kazys, Ultragarsas 63(3), 15 (2008)

3. P. He, IEEE Trans. Ultrason., Fer., Freq. Contr. 45(1), 114 (1998)

4. F. Reed, T. Batzinger, R. Reed, Rev. of Pr. in QNE 12, 1265 (1993)

5. G. Herman, Fundamentals of computerized tomography: Image reconstruction from projection (Springer-Verlag London, 2009)

6. R. Rausutis, R. Kazys, L. Mazeika, NDT\&I 40, 324 (2007)

7. R. Rausutis, R. Kazys, L. Mazeika, NDT\&I 41, 300 (2008)

8. K. Kvasnikov, A. Soldatov, I. Bolotina, M. Krening, A. Potapenko, Rus. J. NDT 49(11), 625 (2013)

9. E. Magrab, An Engineer's Guide to Mathematica (John Wiley \& Sons, 2014)

10. D. Buy, V. Makarov, Al. Soldatov, An. Soldatov, Intern. J. A\&B. Res. 12, 216 (2015)

11. A. Goncharsky, S. Romanov, S. Seryozhnikov, WM 51(3) 389 (2014)

12. S. Deans, The Radon Transform and Some of Its Applications (DP, N.Y. 31 East 2-nd str., Mineola, 2007) 